\title{
AC conductivity and broadband dielectric spectroscopy of a poly(vinyl chloride)/poly(ethyl methacrylate) polymer blend
}

\author{
T FAHMY ${ }^{1,2, *}$ (1) and HESHAM ELZANATY ${ }^{3}$ \\ ${ }^{1}$ Plasma Technology and Material Science Unit (PTMSU), Physics Department, College of Science and Humanity, Prince \\ Sattam Bin Abdulaziz University (PSAU), Al Kharj 11942, Kingdom of Saudi Arabia \\ ${ }^{2}$ Polymer Research Group, Physics Department, Faculty of Science, Mansoura University, Mansoura 35516, Egypt \\ ${ }^{3}$ Department of Basic Science, Faculty of Engineering, Delta University, Mansoura 11152, Egypt \\ *Author for correspondence (tfahmy_5@yahoo.com)
}

MS received 20 November 2018; accepted 2 April 2019

\begin{abstract}
Alternating-current (ac) conductivity and dielectric relaxation behaviour of a poly(vinyl chloride)/poly(ethyl methacrylate) polymer blend have been investigated intensively in a frequency range from $1 \times 10^{-1}$ to $2 \times 10^{7} \mathrm{~Hz}$ through a temperature range from 300 to $393 \mathrm{~K}$. The variation of $\sigma_{\mathrm{ac}}$ of pure and polyblend samples showed a plateau region at high temperature and low frequency and this plateau region is decreased with decreasing temperature. Values of the exponent $n$ are less than unity indicative of the correlated barrier hopping for conduction. The values of the exponent $n$ are used to calculate the binding energy $\left(W_{\mathrm{m}}\right)$ of the charge carriers. The investigation of the frequency dependence of $\varepsilon^{\prime}$ for pure and polyblend samples showed a dielectric dispersion. The high values of dielectric constant at a low frequency and high temperature are attributed to the effects of space charge due to the electrode polarization. The complex electric modulus $\left(M^{*}\right)$ of pure and polyblend samples has been investigated. It is found that the real part of the complex electric modulus, $M^{\prime}$ is increased non-linearly as the frequency increased and reached the steady state at higher frequencies for all samples. On the other hand, the imaginary part of the complex electric modulus, $M^{\prime \prime}$ is characterized by a relaxation peak. The different modes of relaxation, such as interfacial polarization and dipolar relaxation, are detected in low and high frequency regions in the variation plot of $M^{\prime \prime}$ against frequency. The activation energy values of both interfacial polarization and $\alpha$-relaxation are calculated.
\end{abstract}

Keywords. AC conductivity; dielectric; electric modulus; interfacial polarization; activation energy.

\section{Introduction}

The aim of mixing polymers with each other is to obtain new products with unique properties at lower cost. The blending process has various economic and property advantages, such as, ability to reduce material cost, tremendous development of modified materials, lightweight and ability to improve the processability of polymeric materials. Generally, polymers and polymer blends have gained more technological importance in fields of electrophotography, optoelectronics, electro-optical devices, shielding, fuel cell, capacitors and nanocomposite structures [1-5].

The chemical structure of poly(ethyl methacrylate) (PEMA) has a methacrylic ester group. Hence, PEMA is characterized by an excellent chemical resistance, high optical transparency and showing interesting properties such as high elasticity and adhesion [6]. PEMA is used as the host polymer and is reported for the first time by Han et al [7] and Fahmy and Ahmed [8-10]. Poly(vinyl chloride) (PVC) is a commercially available inexpensive, corrosion-resistant material and it belongs to the main category of engineering plastics. The chemical and physical properties of PVC can be modified due to its compatibility with a large number of other polymers, such as, PEMA and nitrile rubber $[9,11]$.

The study of dielectric behaviour of the polymers and polymer blends has recently drawn great attention because of their basic and applied aspects [12-14]. The dielectric relaxation is a very sensitive method for investigating the miscibility and compatibility of polymer blends. The measurement of dielectric properties as a function of temperature and frequency is an interesting technique for investigating the reorientation of the dipoles through molecular and group rotation [15]. The thermally stimulated depolarization current technique is used previously to investigate the relaxation phenomenon of the PVC/PEMA polyblend [8-10]. The aim of this study is devoted to investigate alternating current (ac) electrical conductivity and dielectric relaxation spectroscopy of the PVC/PEMA polymer blend.

\section{Experimental}

\subsection{Materials}

PVC and PEMA are obtained from BDH Chemicals Ltd., England and Poly Science, Inc., USA, respectively. 

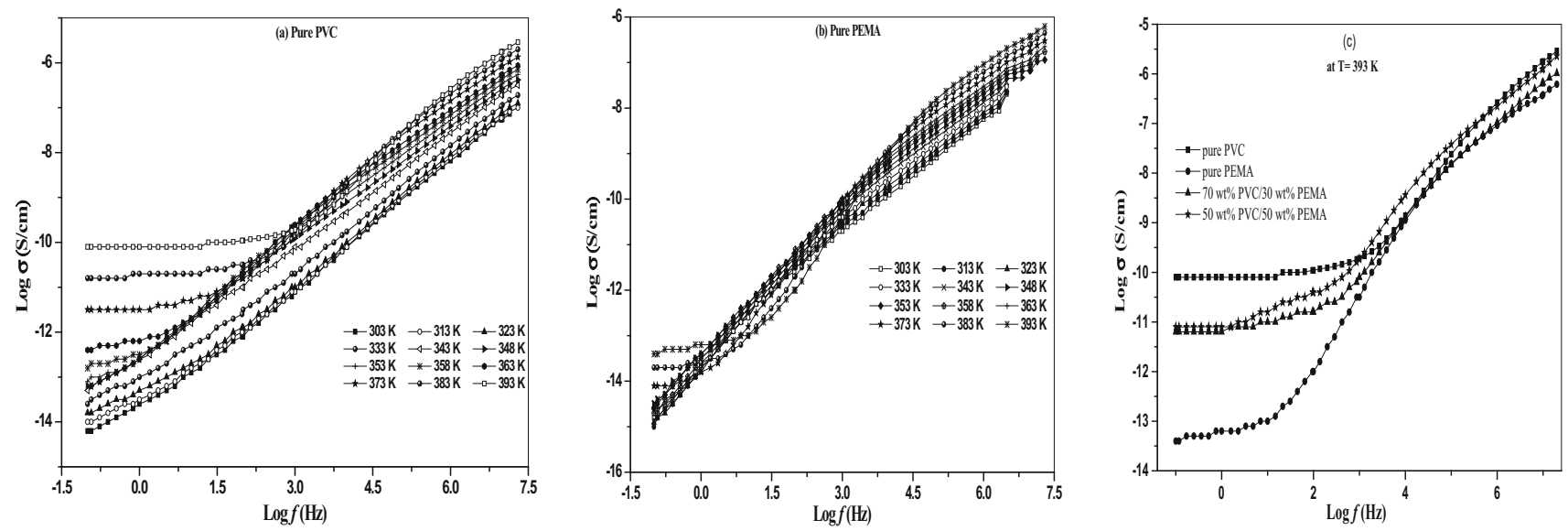

Figure 1. $\log \sigma$ against $\log f$ at fixed temperatures for (a) PVC, (b) PEMA and (c) PVC, PEMA and polyblend samples.

\subsection{Materials preparation}

Tetrahydrofuran (THF) is used as a common solvent for PVC and PEMA. Polyblend samples are prepared by dissolving PVC and PEMA together in THF and stirred for several hours to produce a homogenous solution. Films of the samples are obtained after casting the solution into a glass dish at $323 \mathrm{~K}$ for two days in an oven to ensure that the solvent is evaporated completely from the films.

Measurements of ac conductivity and dielectric are carried out in a wide range of frequency from $1 \times 10^{-1}$ to $20 \times 10^{6} \mathrm{~Hz}$ through a range of temperature from 298 to $393 \mathrm{~K}$ by broadband dielectric spectroscopy (Novocontrol turnkey concept 40 system) at the national research centre, NRC, Egypt. The temperature is controlled by the QUATRO Cryosystem.

\section{Results}

\subsection{AC conductivity}

Variation of ac conductivity, $\sigma_{\mathrm{ac}}$, against frequency for PVC, PEMA and its polyblend samples at different fixed temperatures is displayed in figure $1 \mathrm{a}-\mathrm{c}$. One can observe that the conductivity has strong dependence on frequency and temperature due to the insulating nature of the samples. Electrical conductivity values, especially for $50 \mathrm{wt} \% \mathrm{PVC} / 50 \mathrm{wt} \%$ PEMA, are found to be different from previously published. This is due to several factors, such as the method of preparation of materials and measurement conditions [4]. It is found that at very low frequencies ac conductivity values decrease with a decrease in the frequency. This decreasing in $\sigma_{\mathrm{ac}}$ is due to the dipole polarization or Maxwell-Wagner-Sillars (MWS) interfacial polarization. Depending on MWS polarization, the interfaces between the segments of PVC and PEMA behave like potential barriers and the charge carriers within the segment of the polymers will behave like charges in potential well. At a low frequency, there is a possibility for some of the

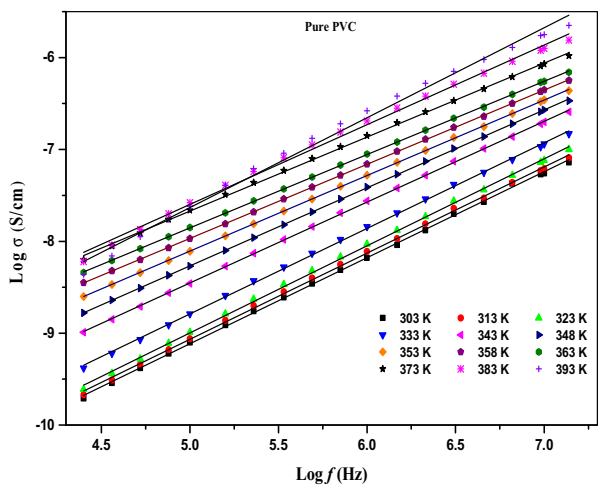

Figure 2. $\log \sigma$ against $\log f$ at fixed temperatures for pure PVC.

charge carriers to tunnel between the segments, resulting in small electrical conductivity. With an increase in frequency, the tunnelling possibility of a large number of charge carriers will take place leading to an increase in electrical conductivity. At a high frequency, the charge carriers will have enough energy to overcome the potential barrier, leading to a significant increase in electrical conductivity. On the other hand, the variation of $\sigma_{\mathrm{ac}}$ of pure and polyblend samples displays a plateau region at high temperature and in a low frequency region as shown in figure $1 \mathrm{c}$. With a decrease in temperature, the plateau region is decreased and $\sigma_{\mathrm{ac}}$ is found to be highly dependent on frequency. The general behaviour of the $\sigma_{\mathrm{ac}}$ is that of the universal power law [16]

$$
\sigma_{\mathrm{ac}}=\sigma_{\mathrm{dc}}+A \omega^{n}
$$

where $\sigma_{\mathrm{ac}}, \sigma_{\mathrm{dc}}, A$ and $n$ are the real part of electrical conductivity, direct current (dc) conductivity, a constant depends on temperature and an exponent, respectively. It is found that equation (1) can not fit the experimental data in the whole range of frequency. Values of the exponent $n$ for all samples have been estimated from the slope of $\log \sigma$ against $\log f$ at higher frequencies at fixed temperatures as shown in figure 2 
Table 1. Values of both $n$ and $W_{\mathrm{m}}$ for all samples.

\begin{tabular}{|c|c|c|c|c|c|c|c|c|}
\hline \multirow[b]{2}{*}{$T(\mathrm{~K})$} & \multicolumn{2}{|c|}{ Pure PVC } & \multicolumn{2}{|c|}{ Pure PEMA } & \multicolumn{2}{|c|}{$70 \mathrm{wt} \% \mathrm{PVC}$} & \multicolumn{2}{|c|}{$50 \mathrm{wt} \% \mathrm{PVC}$} \\
\hline & $n$ & $W_{\mathrm{m}}(\mathrm{eV})$ & $n$ & $W_{\mathrm{m}}(\mathrm{eV})$ & $n$ & $W_{\mathrm{m}}(\mathrm{eV})$ & $n$ & $W_{\mathrm{m}}(\mathrm{eV})$ \\
\hline 303 & 0.935 & 2.43 & 0.566 & 0.361 & 0.795 & 0.765 & 0.854 & 1.073 \\
\hline 313 & 0.918 & 1.98 & 0.717 & 0.572 & 0.859 & 1.152 & 0.828 & 0.944 \\
\hline 323 & 0.929 & 2.35 & 0.621 & 0.441 & 0.884 & 1.443 & 0.869 & 1.276 \\
\hline 333 & 0.916 & 2.05 & 0.579 & 0.409 & 0.902 & 1.769 & 0.868 & 1.312 \\
\hline 343 & 0.871 & 1.38 & 0.609 & 0.454 & 0.856 & 1.236 & 0.857 & 1.244 \\
\hline 348 & 0.843 & 1.14 & 0.642 & 0.503 & 0.853 & 1.225 & 0.843 & 1.150 \\
\hline 353 & 0.818 & 1.00 & 0.674 & 0.560 & 0.846 & 1.192 & 0.838 & 1.128 \\
\hline 358 & 0.803 & 0.94 & 0.689 & 0.596 & 0.839 & 1.150 & 0.819 & 1.022 \\
\hline 363 & 0.793 & 0.91 & 0.694 & 0.614 & 0.826 & 1.083 & 0.796 & 0.921 \\
\hline 373 & 0.801 & 0.96 & 0.698 & 0.639 & 0.804 & 0.986 & 0.775 & 0.858 \\
\hline 383 & 0.859 & 1.40 & 0.714 & 0.693 & 0.808 & 1.031 & 0.768 & 0.856 \\
\hline 393 & 0.964 & 5.64 & 0.758 & 0.842 & 0.846 & 1.326 & 0.795 & 0.994 \\
\hline
\end{tabular}

and listed in table 1. It is found that values of the exponent $n$ of all samples are less than unity indicative of the correlated barrier hopping $(\mathrm{CBH})$ for conduction [17-22].

The variation of $n$ values is indication of the change in the nature of the conduction process depending on the change in blending and temperature. The values of $n$ are used to calculate binding energy values $\left(W_{\mathrm{m}}\right)$ of charge carriers using the empirical formula, $W_{\mathrm{m}}=6 k_{\mathrm{B}} T /(1-n)[23,24] . W_{\mathrm{m}}$ is known as the difference in energy of both the ground state of the potential well and ionized state. Values of $W_{\mathrm{m}}$ have been determined and tabulated in table 1.

It is useful to analyse the results of ac conductivity in the whole range of frequency to obtain more valuable information about the conductivity mechanism of pure and polyblend samples around glass transition temperature $\left(T_{\mathrm{g}}\right)$. Figure 3 shows the Arrhenius plots of $\log \sigma$ against $1 / T$ at fixed frequencies for pure and polyblend samples, according to the following equation:

$$
\sigma=\sigma_{0} \exp \left(-\frac{E_{\mathrm{a}}}{k_{\mathrm{B}} T}\right) \text {. }
$$

Two regions in these plots with different slopes around $T_{\mathrm{g}}$ are obtained. These results suggest that there is a change in the conduction mechanism in the vicinity of glass transition temperature of both PVC and PEMA, from 338 to $357 \mathrm{~K}$, where $T_{\mathrm{g}}$ of pure PEMA and PVC is 344 and $357 \mathrm{~K}$, respectively [10]. Activation energy $\left(E_{\mathrm{a}}\right)$ values are determined as a function of frequency and summarized in table 2 . The activation energy values of pure and polyblend samples are found to be frequency dependent and they decrease with increasing frequency.

\subsection{Dielectric spectra}

Analysis of the dielectric is a useful method to study the molecular interaction in polymer blends. The complex dielectric permittivity $\left(\varepsilon^{*}\right)$ is written as follows:

$$
\varepsilon^{*}=\varepsilon^{\prime}-j \varepsilon^{\prime \prime},
$$

where $\varepsilon^{\prime}$ (dielectric constant) and $\varepsilon^{\prime \prime}$ (dielectric loss) are the real value and imaginary value of complex dielectric permittivity. Figure 4 illustrates variation of $\varepsilon^{\prime}$ for pure PVC, PEMA and polyblend samples with the frequency at different temperatures. As already known, the permittivity of the polymeric material arises due to the polarization of the molecules. At a lower frequency the mobile charges have the sufficient time to propagate under the action of the applied external electric field leading to charge accumulation at the polymer electrode interface. Since, the charges can not transport to the external circuit, they will accumulate at the polymer electrode interface forming a heterocharge layer [17]. If the thickness of the heterocharge layer is lower than the polymer thickness, the charge density will increase quickly resulting in electrode polarization [18]. Thus, the effects of space charge due to the electrode polarization lead to an increase in the values of dielectric constant at a lower frequency. On the other hand, at a higher frequency, the periodic reversal of the applied electric field occurred rapidly and there is no sufficient time to accumulate the charges. Therefore, the polarization due to the accumulation of charges will decrease resulting in a decrease in the dielectric constant. Generally, dielectric constant, $\varepsilon^{\prime}$, of the dielectric materials decreases as the frequency increases and this behaviour is interpreted by dielectric relaxation.

The change in dielectric constant with temperature at a frequency of $\sim 0.1 \mathrm{~Hz}$ is shown in figure 5. This frequency is chosen to avoid the conductivity effects which block the behaviour of the dipoles at high temperature and low frequency. One can observe that the dielectric constant is low at the temperatures lower than glass transition temperature of the samples. At the low temperatures and constant frequency, the orientation of the dipoles does not obey the electric field because of the high viscosity of the polymer leading to a 

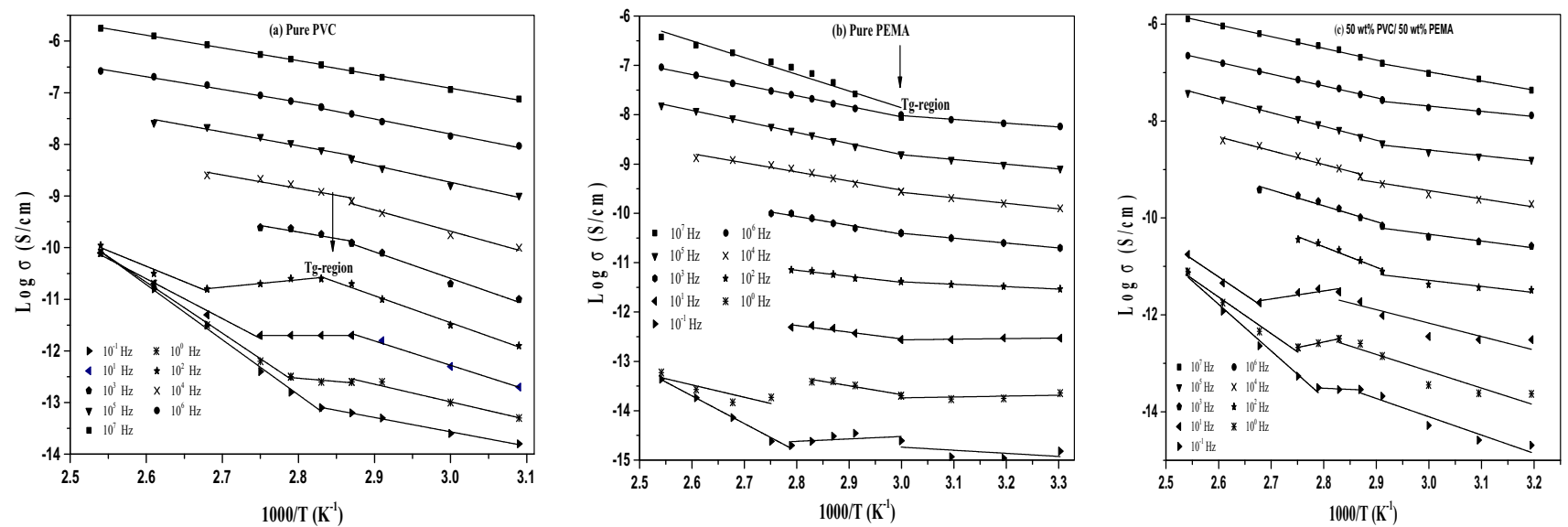

Figure 3. $\log \sigma$ against $1000 / T$ for (a) PVC, (b) PEMA and (c) $50 \mathrm{wt} \%$ PVC/50 wt $\%$ PEMA.

Table 2. Activation energy values of pure and polyblend samples at different frequencies.

\begin{tabular}{|c|c|c|c|c|c|c|c|c|}
\hline \multirow[b]{2}{*}{$F(\mathrm{~Hz})$} & \multicolumn{2}{|c|}{ Pure PVC } & \multicolumn{2}{|c|}{ Pure PEMA } & \multicolumn{2}{|c|}{$70 \mathrm{wt} \% \mathrm{PVC}$} & \multicolumn{2}{|c|}{$50 \mathrm{wt} \% \mathrm{PVC}$} \\
\hline & $E_{\mathrm{a} 1}(\mathrm{eV})$ & $E_{\mathrm{a} 2}(\mathrm{eV})$ & $E_{\mathrm{a} 1}(\mathrm{eV})$ & $E_{\mathrm{a} 2}(\mathrm{eV})$ & $E_{\mathrm{a} 1}(\mathrm{eV})$ & $E_{\mathrm{a} 2}(\mathrm{eV})$ & $E_{\mathrm{a} 1}(\mathrm{eV})$ & $E_{\mathrm{a} 2}(\mathrm{eV})$ \\
\hline 0.1 & 0.23 & 0.91 & 0.05 & 0.48 & 0.26 & 0.91 & 0.32 & 0.82 \\
\hline 1 & 0.30 & 0.85 & 0.02 & 0.22 & 0.37 & 0.74 & 0.30 & 0.65 \\
\hline 10 & 0.41 & 0.66 & 0.01 & 0.12 & 0.05 & 0.39 & 0.24 & 0.62 \\
\hline 100 & 0.45 & 0.51 & 0.04 & 0.11 & 0.06 & 0.41 & 0.11 & 0.36 \\
\hline 1000 & 0.44 & 0.22 & 0.08 & 0.15 & 0.05 & 0.35 & 0.12 & 0.28 \\
\hline 10,000 & 0.35 & 0.22 & 0.10 & 0.16 & 0.07 & 0.32 & 0.15 & 0.25 \\
\hline 100,000 & 0.28 & 0.23 & 0.08 & 0.19 & 0.06 & 0.28 & 0.10 & 0.24 \\
\hline $1,000,000$ & 0.25 & 0.21 & 0.06 & 0.18 & 0.07 & 0.25 & 0.09 & 0.21 \\
\hline $10,000,000$ & 0.22 & 0.21 & - & 0.29 & 0.12 & 0.24 & 0.16 & 0.20 \\
\hline
\end{tabular}
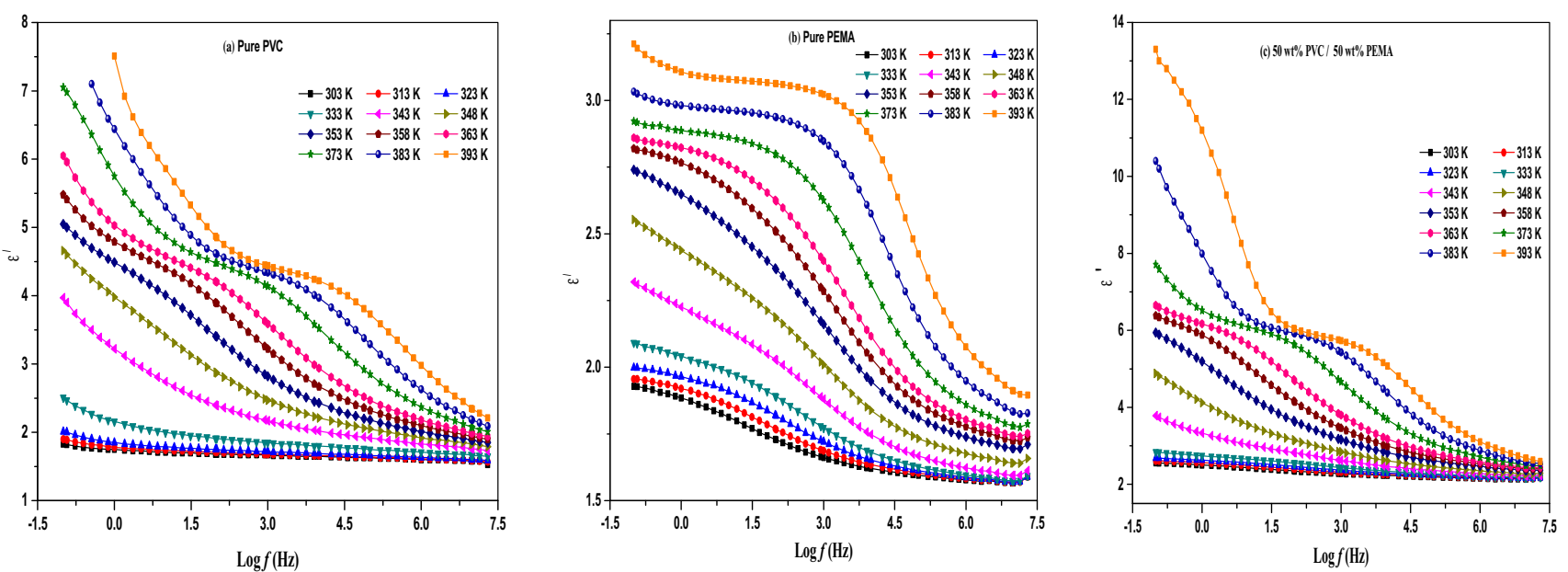

Figure 4. The variation of $\varepsilon^{\prime}$ against $\log f$ for (a) PVC, (b) PEMA and (c) $50 \mathrm{wt} \%$ PVC/50 wt $\%$ PEMA. 


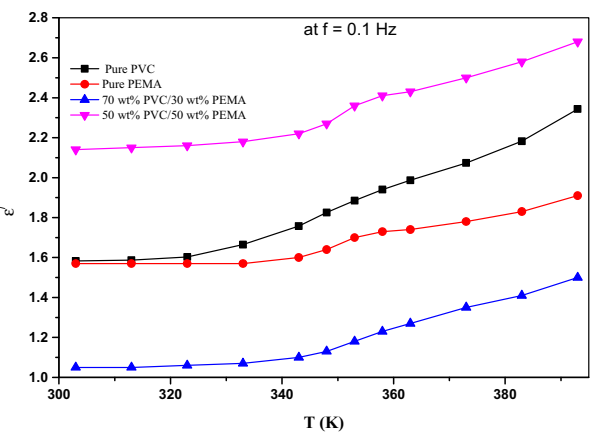

Figure 5. Dielectric constant, $\varepsilon^{\prime}$, vs. temperature at a fixed frequency.

lower dielectric constant. On the other hand, with increase in temperature, viscosity of the samples will decrease slightly and facilitate the dipole orientation with the field, which increases the dielectric constant values.

\subsection{Electric modulus part}

Investigation of the electric modulus is another method for analysing dielectric relaxation behaviour of polymeric materials. The complex electric modulus $M^{*}(\omega)$ can be obtained using complex permittivity $\varepsilon^{*}(\omega)$ from the following equation, $M^{*}(\omega)=1 / \varepsilon^{*}(\omega)$. The components of complex dielectric permittivity, $\varepsilon^{*}(\omega)$, are converted to the components of complex electric modulus, $M^{*}(\omega)$, as follows:

$$
\begin{aligned}
& M^{*}=M^{\prime}+j M^{\prime \prime}, \quad j=\sqrt{-1} \\
& M^{\prime}=\frac{\varepsilon^{\prime}}{\varepsilon^{\prime 2}+\varepsilon^{\prime \prime 2}}, \quad M^{\prime \prime}=\frac{\varepsilon^{\prime \prime}}{\varepsilon^{\prime 2}+\varepsilon^{\prime \prime 2}} .
\end{aligned}
$$

The explanation of relaxation behaviour using the complex electric modulus formalism gives more advantages upon other treatments, since large variations in the dielectric permittivity and dielectric loss at lower frequency and higher temperature
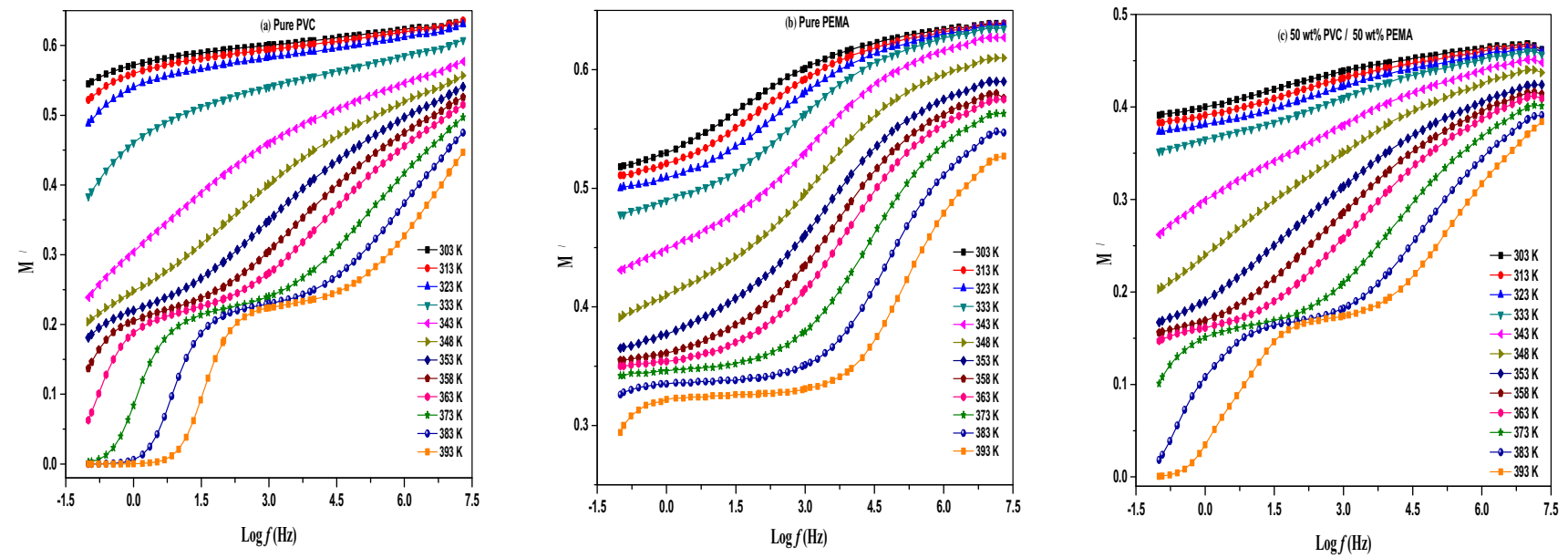

Figure 6. $M^{\prime}$ against $\log f$ at fixed temperature for (a) PVC, (b) PEMA and (c) $50 \mathrm{wt} \%$ PVC/50 wt $\%$ PEMA.
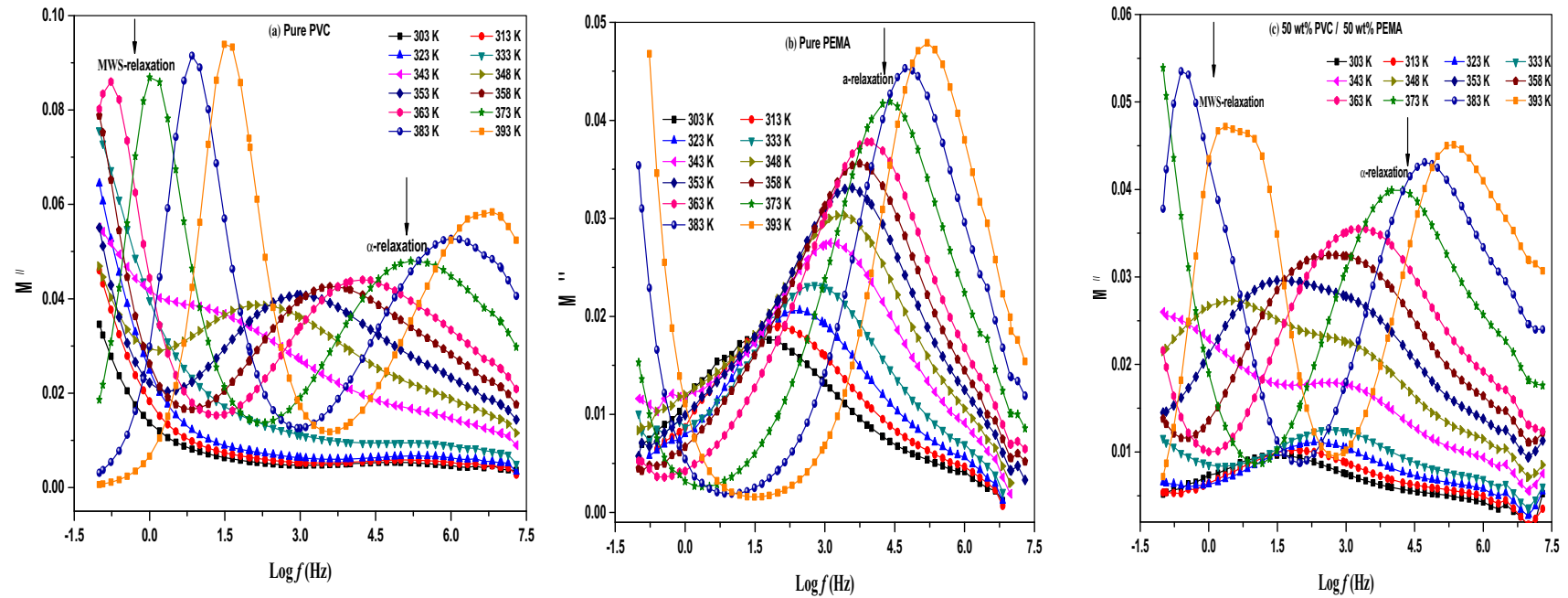

Figure 7. $M^{\prime \prime}$ against $\log f$ at fixed temperature for (a) PVC, (b) PEMA and (c) $50 \mathrm{wt} \%$ PVC/50 wt $\%$ PEMA. 


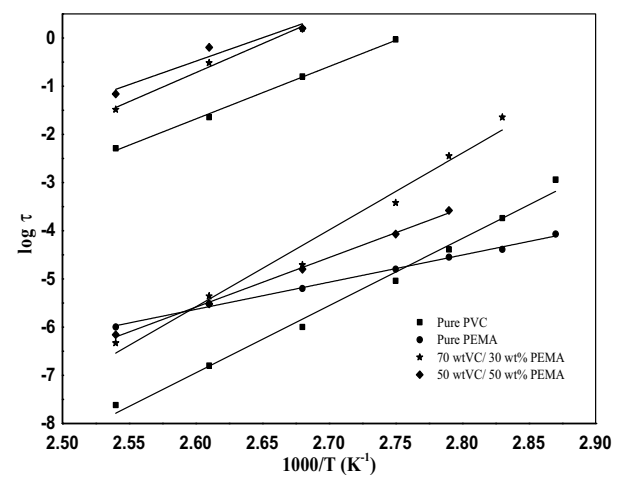

Figure 8. $\log \tau$ vs. 1000/T for all samples.

Table 3. Activation energy values of both interfacial polarization and $\alpha$-relaxation.

\begin{tabular}{lcc}
\hline & \multicolumn{2}{c}{$E_{\mathrm{a}}(\mathrm{eV})$} \\
\cline { 2 - 3 } Sample & $\begin{array}{c}\text { Interfacial } \\
\text { polarization }\end{array}$ & $\alpha$-Relaxation \\
\hline Pure PVC & 0.93 & 1.20 \\
$70 \mathrm{wt} \%$ PVC/30 wt\% PEMA & 1.02 & 1.29 \\
$50 \mathrm{wt} \%$ PVC/50 wt\% PEMA & 0.83 & 1.03 \\
PEMA & - & 0.49 \\
\hline
\end{tabular}

are minimized. Also, all difficulties due to electrode nature, electrode-sample contact and space charge injection can be ignored. The change in both real and imaginary parts of the complex electric modulus, $M^{\prime}$ and $M^{\prime \prime}$, for PVC, PEMA and $50 \mathrm{wt} \%$ PVC sample, as a representative sample of polyblend samples is shown in figures 6 and 7. One can observe that the real part of the complex electric modulus, $M^{\prime}$ increases non-linearly as the frequency increases and finally reaches the steady state at higher frequencies.

On the other hand, it is observed that the imaginary part of the complex electric modulus, $M^{\prime \prime}$ spectra of pure PVC and PVC/PEMA blends is characterized by two different relaxation peaks in lower and higher frequency regimes, while, $M^{\prime \prime}$ spectra of PEMA are characterized by one relaxation peak and are detected in the higher frequency region. The lower frequency relaxation peak can be attributed to the interfacial polarization or MWS polarization process [25], whereas, the higher frequency region peak is attributed to the cooperative chain segmental motion, i.e., $\alpha$-relaxation, $\mathrm{C}-\mathrm{Cl}$ dipole orientation in the case of PVC and free rotation of the ethyl ester group attached to the polymer chain in PEMA.

Since the electrode polarization is ignored in electric modulus formalism [26] and electrical conductivity of the samples is low, the slower process could unambiguously be related to interfacial polarization. The interfacial polarization is characterizing heterogeneous materials because of the charge accumulation at the interfaces leading to the formation of large dipoles with greater relaxation time, which try to obey the alternation of the applied electric field. The broadness of $M^{\prime \prime}$ for polyblend samples is due to the distribution of relaxation time because of the inhomogeneous behaviour of the relaxing dipoles of polyblend samples. Generally, a noticeable shift in the position of the relaxation peak to a higher frequency side with increasing temperature has been observed for pure and polyblend samples as shown in figure 7 . This shift in the peak position with increasing temperature corresponds to the conductivity current relaxation.

The maximum frequency $f_{\max }$ values of $M^{\prime \prime}$ plots are used to calculate the characteristic relaxation time $\tau, \tau=$ $1 / 2 \pi f_{\max }$. Figure 8 displays a linear behaviour between $\log \tau$ and $1 / T$ according to the Arrhenius equation:

$$
\tau=\tau_{0} \exp \left(\frac{E_{\mathrm{a}}}{k_{\mathrm{B}} T}\right)
$$

where $\tau_{0}, E_{\mathrm{a}}, k_{\mathrm{B}}$ and $T$ are the pre-exponential factor, activation energy, Boltzmann's constant and temperature, respectively. The relaxation time is decreasing with increasing temperature of the samples, where the dissipated thermal energy assisted the formed dipoles to follow the motion of the alternating electric field. Hence, by knowing the slope, the activation energy values of both interfacial polarization and $\alpha$-relaxation are determined and summarized in table 3.

\section{Conclusion}

Films of PVC/PEMA with different concentrations are prepared using a casting method and ac electrical conductivity, dielectric relaxation behaviour and electric modulus of all samples are studied in the range of frequency from $1 \times 10^{-1}$ to $2 \times 10^{7} \mathrm{~Hz}$ at different temperatures ranging from 300 to $393 \mathrm{~K}$. It is observed that ac electrical conductivity is very low at lower frequencies and it increased with increasing frequency and temperature because of the increase in charge carrier mobility. The values of the exponent $n$ are less than unity indicative of the $\mathrm{CBH}$ for conduction. The investigation of the frequency dependence of $\varepsilon^{\prime}$ for pure and polyblend samples showed a dielectric dispersion. Interfacial polarization and $\alpha$-relaxation are detected in low and high frequency regions in the variation plot of $M^{\prime \prime}$ against frequency. It is found that the dielectric relaxation time is decreased with increasing temperature which is estimated from the variation of the imaginary part of the electric modulus, $M^{\prime \prime}$ against frequency. The activation energy values of both interfacial polarization and $\alpha$-relaxation are calculated.

\section{References}

[1] Balart R, Lopez J, Garcia D and Salvador M D 2005 Eur. Polym. J. 412150 
[2] Qi R, Chen Z and Zhou C 2005 Polymer 464098

[3] Patil D S, Shaikh J S, Dalavi D S, Kalagi S S and Patil P S 2011 Mater. Chem. Phys. 128449

[4] Mohan K R, Achari V B S, Rao V V R N and Sharma A K 2011 Polym. Test. 30881

[5] Kowalonek J 2016 Polym. Deg. Stab. 133367

[6] Sim L N, Majid S R and Arof A K 2012 Vib. Spectrosc. 58 57

[7] Han H S, Kang H R, Kim S W and Kim H T 2002 J. Power Sources 112461

[8] Fahmy T and Ahmed M T 2001 Polym. Test. 20477

[9] Fahmy T and Ahmed M T 2000 Polym. Int. 49669

[10] Fahmy $\mathrm{T}$ and Ahmed M T 1999 Polym. Test. 18 589

[11] Migahed M D, Ishra M, El-Khodary A and Fahmy T 1993 Polym. Test. 12335

[12] Fahmy T and Ahmed M T 2011 J. Korean Phys. Soc. 58 1654

[13] Fahmy T 2007 Polym. Plast. Technol. Eng. 467

[14] Migahed M D, Ishra M, Fahmy T and Barakat A 2004 J. Phys. Chem. Solids $\mathbf{6 5} 1121$
[15] Shukla J P, Manohar R, Shukla P and Gupta M 1999 Proceedings of 13th International Conference on Dielectric Liquids (ICDL '99), Nm, Japan, July 20-25

[16] Jonscher A K 1977 Nature 267673

[17] Woo H J, Majid S R and Arof A K 2012 Mater. Chem. Phys. 134 755

[18] Howell F S, Bose R A, Macedo P B and Moynihan C T 1974 J. Phys. Chem. 78639

[19] Paquin L, St-Onge H and Wertheimer M R 1982 IEEE Trans. Electr. Insul. EI-17 399

[20] Fahmy T 2001 J. Polym. Mater. 50109

[21] Jonscher A K 1990 IEEE Electr. Insul. 616

[22] More S, Dhokne R and Moharil S 2017 Mater. Res. Express 4 055302

[23] Pike G E 1972 Phys. Rev. B 61572

[24] Jung W H 2000 J. Phys. D: Appl. Phys. 33444

[25] Passaras G C, Gatos K G, Karahaliou P K, Georga S N, Krontiras C A and Krger-Kocsis J 2007 Express Polym. Lett. 1 837

[26] Psarras G C, Manolakaki E and Tsangaris G M 2003 Compos. A: Appl. Sci. Manuf. $\mathbf{3 4} 1187$ 